\title{
Understanding of the Vortex Lattice Behavior Induced by Selective Removal of Atoms from a Bose-Einstein Condensate
}

\author{
Hui Zhai and Qi Zhou \\ Center for Advanced Study, Tsinghua University, Beijing, China
}

\begin{abstract}
.
It is known that the quantized vortices in a superfluid can be described by a dual electromagnetic model through the duality transformation. Recently a new technique, which can selectively remove atoms from a Bose-Einstein condensate, was applied to the vortex lattice state of the rapid rotating Boson gases. The increase of vortex number, dynamical formation of giant vortex, the oscillation and damping of vortex motion were observed in these experiments. In this paper we will discuss these observations in the framework of dual description and show how to understood these observations naturally.
\end{abstract}




\section{Introduction}

It is well known that quantized vortices play an important role in understanding many phenomena of superfluid, superconductivity and even astrophysics. In the latest several years a remarkable experimental development has been made in the rapidly rotating Bose-Einstein condensate(BEC). A large amount of angular momentum is imparted into the system and its rotation frequency becomes faster and faster. The ground state of the fast rotating BEC was observed to be a regular triangular vortex lattice at nearly zero temperature. This provides a new stage of studying both the equilibrium and the dynamic properties of the vortex matter.

Recently two novel experiments were reported by Cornell's group in JILA. [1 2] Atoms in the hyperfine spin $|F=1\rangle$ state, were rotated rapidly by the evaporative spin-up mechanism. About 180 vortices were observed in the cloud and they were arranged into a regular lattice structure. And then, an atom removal laser, whose frequency was tuned to the transition energy between the $|F=1\rangle$ state and the $|F=0\rangle$ state, was applied to the center of the condensate, and the recoil from a spontaneously scatted photon blasts atoms out of the condensate. In this way, the atoms with lower angular momentum were selectively removed from the system.

Many exotic features were observed when this technique is applied. After the atom removal laser was applied continuously, the vortex number contained in the cloud increased form 180 to about 230, and the vortices began to aggregate to the center of the condensate, eventually they formed a giant vortex. If the laser was applied for a short limited time, it was reported in Ref.1 that a periodic formation and disappearance of the giant vortex core was observed after applying a strong atom removal pulse, and in Ref. 2] that the Tkachenko oscillation of the vortex lattice would be found after a weak pulse. Both kinds oscillation were found damped. [1] 2]

A number of questions are raised from these experiments, such as how to understand the vortex number increase and the vortices aggregating to the center of condensate, why the strong and weak laser strength will cause two different kinds of oscillations, how to understand the relation between the oscillation frequency and the initial conditions of the condensate, and which mechanism leads to the damping. We notice that recently there are lots of theoretical efforts around these experiments, some of them try to understand these observations numerically [3] and some of them focus on the Tkachenko wave of the vortex lattice state 44 [5] 6]. However, in this paper, we will present an explanation to these questions from another point of view, namely using a dual 2+1-dimensional Maxwell electrodynamic (MED) description, and we will show that the main features observed can be understood naturally in this framework, although qualitatively in some aspects.

This dual electromagnetic description of the interacting bosons model was obtained by the duality transformation early in the study of Helium superfluidity, [ $[9]$ and then in the past decades it has been discussed in the contents of superconductivity and superfluid film by various authors. 10] 11] Compared to the model discussed before, we would like to emphasize some important differences of the system studied here. First, a large amount of angular momentum have been imparted into the atoms and the rotational invariance is restored before the formation of condensate, therefore the total angular momentum of the condensate should be conserved to a non-zero value. The second, the atom removal laser violates the current conservation condition. And the third, the system is dilute and the interaction between atoms is weak, the sound velocity is consequently relatively small. We will show that these differences 
lead to these exotic phenomena in these experiments, in other words, these observed phenomena reflect some intrinsic properties of the rotating BEC.

\section{The Dual Picture}

In this section we will firstly make a brief description of the dual picture for the interacting bosons system, for the details we refer to the Ref. [9] [10] [1]. Beginning with the coherent state path integral formulism, [12] we write down the quantum mechanical propagator

$$
Z=\int D[\psi, \bar{\psi}] \exp \left\{i \int d t d^{2} \vec{r} \mathcal{L}\right\}
$$

where the Lagrangian

$$
\mathcal{L}=-\hbar \bar{\psi} \frac{\partial_{t}}{i} \psi-\frac{\hbar^{2}}{2 m}\left|\frac{\vec{\partial}}{i} \psi(r)\right|^{2}-\frac{g}{2}|\psi(r)|^{4} .
$$

$g$ is the interaction strength and equals to $4 \pi \hbar^{2} a_{s c} / m$ for ultracold atomic system, where $a_{s c}$ is the $s$-wave scattering length between atoms. Here $\psi$ is a complex field describing the bosonic many-body system, and $\bar{\psi}$ is its complex conjecture. We can factor $\psi$ as $\sqrt{\rho} e^{i \theta} e^{i \theta_{v}}$. The field $\rho$ describes the density of bosons. The phase of $\psi$ contains two parts, $\theta$ is the smooth part free from singularity and $\theta_{v}$ is that caused by the presence of vortices.

First of all, we can introduce an auxiliary field $\vec{J}$ by performing a HubbardStratanovich (H-S) transformation, the physical meaning of which is the superfluid current. By defining the three vectors $\tilde{J} \equiv(\hbar \rho, \hbar \vec{J})$ and $\tilde{\nabla}=\left(\partial_{t}, \vec{\nabla}\right)$, in the low frequency and long wavelength limits one can neglect the second order gradient term $|\nabla \rho|^{2} / \rho$, and obtain that

$$
Z=\int D[J] D[\rho] D[\theta] D\left[\theta_{v}\right] \exp \left\{i \int d t d^{2} \vec{r}\left(-\tilde{J} \cdot \tilde{\nabla} \theta-\tilde{J} \cdot \tilde{\nabla} \theta_{v}+\frac{m}{2 \rho}|\vec{J}|^{2}-\frac{g}{2} \rho^{2}\right)\right\} .
$$

Integrating over $\theta$, one can naturally obtain a $\delta$-function constraint $\tilde{\nabla} \cdot \tilde{J}=0$, which is just the conventional current conservation condition

$$
\frac{\partial \rho}{\partial t}+\vec{\nabla} \cdot \vec{J}=0
$$

Owing to this constraint, we can introduce a three-component gauge field $\tilde{b}$ defined as $\left(b_{0}, \vec{b}\right)$, which satisfies

$$
\tilde{J}=\tilde{\nabla} \times \tilde{b},
$$

the density and the current of the bosons are thereby determined by the gauge field. Hence, $\rho=\vec{\nabla} \times \vec{b}$ is compared to a magnetic field perpendicular to the 2-dimensional plane. We can expand $\rho$ as $\bar{\rho}+\delta \rho(\vec{r}, t), \bar{\rho}$ is the average density which is compared to an external magnetic field, while $\delta \rho(\vec{r}, t)$ is the density fluctuation which is compared to the electromagnetic wave. The electric field $\vec{E}$ is defined as $\vec{\nabla} b_{0}-\partial_{0} \vec{b}$, and it is perpendicular to the boson current $\vec{J}$. Therefore the Eq. (4) can be rewritten as

$$
\frac{\partial B}{\partial t}+\vec{\nabla} \times \vec{E}=0
$$

which is just the Maxwell equation in a monopole free space-time. 
With replacing the $\rho$ by $\bar{\rho}$ in the denominator, the last two terms in Eq.(3) can also be reexpressed in terms of the gauge field $\tilde{b}$, which is just the dynamic term $-F^{\mu \nu} F_{\mu \nu}$ of the gauge field as in the conventional MED. It determines the dynamic properties of boson density fluctuation, i.e. phonon, and recovers the well-known result for the sound velocity $s=\sqrt{g \bar{\rho} / m}$, which plays the same role as the light velocity in $2+1$-dimensional MED.

The coupling between the vortices and bosons can be illustrated as

$$
-\tilde{J} \cdot \tilde{\nabla} \theta_{v}=-\tilde{b} \cdot\left(\tilde{\nabla} \times \tilde{\nabla} \theta_{v}\right)=-\tilde{b} \cdot \tilde{J}_{v}
$$

Here $\tilde{J}_{v}$ is defined as $\tilde{\nabla} \times \tilde{\nabla} \theta_{v}$. It can be verified that the zero-component of $\tilde{J}_{v}$, which is denoted by $\rho_{v}$, represents the vortex density, and the other two spatial components represent the vortex current. The Eq.(7) indicates that the vortices can be viewed as charged particles, which are coupled to an electromagnetic field via gauge coupling. The coupling constant, that is the charge $q$ of the charged particles, is unit. In the Coulomb gauge, the scalar potential $b_{0}$ is instantaneous, one can integrate it out and obtain the mass of vortex $m_{v}$, which is equal to $\bar{\rho} \hbar^{2} \ln \frac{R}{\Lambda} /\left(4 \pi s^{2} m\right)$ for a uniform condensate, and the mutual logarithmic interaction between vortices described by

$$
\mathcal{L}_{i n t}=-\frac{\bar{\rho} \hbar^{2}}{4 \pi m} \int d^{2} \vec{r} d^{2} \overrightarrow{r^{\prime}} \rho_{v}(\vec{r}) \ln \frac{\left|\vec{r}-\overrightarrow{r^{\prime}}\right|}{\Lambda} \rho_{v}\left(\overrightarrow{r^{\prime}}\right) .
$$

Here $R$ is the radius of the condensate and $\Lambda$ is the radius of vortex core.

Thus we have obtained a dual electrodynamic description of quantized vortices, which is briefly summarized in the following table. In the dual picture the vortex lattice state corresponds to the Wigner crystal state of 2-dimensional electrons in the external magnetic field.

\begin{tabular}{|c|c|}
\hline Original Picture & Dual Picture \\
\hline vortex & charged particle \\
\hline $\begin{array}{c}\text { average } \\
\text { superfluid density } \bar{\rho}\end{array}$ & external magnetic field \\
\hline superfluid current $J$ & electric field $E_{\mu}=\epsilon_{\mu \nu} J_{\nu}$ \\
\hline density fluctuation & electromagnetic wave \\
\hline sound velocity & light velocity \\
\hline $\begin{array}{c}\text { the coupling between vortex } \\
\text { and sound wave }\end{array}$ & gauge coupling \\
\hline
\end{tabular}

Such a description can be directly applied to the confined BEC when it is in the Thomas-Fermi regime. The average density here is therefore determined by the trapping potential, as well as the centrifugal repulsive force caused by rotating, and we will take it as inverted parabola in this regime. Here we would like to point out that the main assumption leading to the electrodynamic description is that the vortices are treated as point-like particles, the physics inside the vortex core is not considered, as a consequence we take the mass of vortex as its electromagnetic mass throughout this paper [1]. Besides, in the following treatment we view the system as quasi-two dimensional and neglect the density inhomogeneity when estimating the vortex mass. In the quantum Hall mean field regime [13, it is shown that the physics of vortex core will become important. However, before entering this regime, and when the ThomasFermi theory is still valid, these neglected effects will only slightly renormalize the vortex mass as well as the interaction between vortices. 


\section{Explanation of the Experiments}

\subsection{Vortex Number Increasing}

In JILA experiments the rotational invariance is restored before condensation, therefore the condensate should obey the total angular momentum conservation constraint. Such a system can be described by adding a Lagrange multiplier term to the Lagrangian.

$$
Z=\int d \psi d \Omega \exp \left\{i \int d^{2} \vec{r} d t \mathcal{L}(\psi, \bar{\psi})-\Omega\left(\bar{\psi} \hat{L} \psi-L_{0}\right)\right\} .
$$

It is obvious that one will obtain a $\delta$-function which means that the total angular momentum must always equal to $L_{0}$ after integrating $\Omega$ out. The physical meaning of $\Omega$ is in fact the effective rotation frequency. Following the procedure discussed in above section, it is found that only three additional terms, namely $-2 m b_{0} \Omega+\frac{1}{2} m \Omega^{2} \rho r^{2}+\Omega L_{0}$, will be added to the Lagrangian. One can see that only the zeroth component of the gauge field responds to the rotation. Integrating $\Omega$ out and neglecting the density fluctuation, we obtain

$$
\mathcal{L}=-\frac{\left(-2 m b_{0}+L_{0}\right)^{2}}{2 m\left\langle\rho r^{2}\right\rangle}+\text { other terms. }
$$

Following the same step which leads to Eq.(8), integrating out $b_{0}$, we can obtain a local chemical potential term in the following Lagrangian

$$
\begin{aligned}
& \mathcal{L}_{\text {int }}=-\int d^{2} \vec{r} d^{2} \overrightarrow{r^{\prime}} \rho_{v}(\vec{r}) G\left(\left|\vec{r}-\overrightarrow{r^{\prime}}\right|\right) \rho_{v}\left(\overrightarrow{r^{\prime}}\right)+\int d^{2} \vec{r} \frac{2 L_{0}}{\left\langle\rho r^{2}\right\rangle}\left(\int d^{2} r^{\prime} G\left(\left|\vec{r}-\overrightarrow{r^{\prime}}\right|\right)\right) \rho_{v}(r) \\
& =\mathcal{E}_{\text {int }}+\int d^{2} \vec{r} \mu(\vec{r}) \rho_{v}(\vec{r}) .
\end{aligned}
$$

Here $G\left(\left|\vec{r}-\vec{r}^{\prime}\right|\right)$ is the Green's function for the $b_{0}$ field acquiring a very small mass $2 m /\left\langle\rho r^{2}\right\rangle$, which will vanish in the large atom number limit. The first term in the Eq. (11) indicates that the vortex system can be viewed as an interacting Coulomb gas system, and the second term defines a local chemical potential $\mu(r)$ for vortices emerging from the angular momentum constraint, which is proportional to $L_{0} /\left\langle\rho r^{2}\right\rangle$. The atom removal laser, which removes atoms with lower angular momentum, decreases the atom density $\rho$ with little change to the total angular momentum $L_{0}$, leads to the increase of the vortex chemical potential. Notice that the interaction energy $\mathcal{E}_{i n t}$ depends quadratically dependence on vortex density $\rho_{v}$, and the interaction between vortices are repulsive, so

$$
\frac{\partial \mu}{\partial \rho_{v}}=\frac{\delta^{2} \mathcal{E}_{i n t}}{\delta \rho_{v}^{2}}>0
$$

This shows the increase of vortex density.

\subsection{Aggregate}

For simplicity we assume that the atom removal laser acts only at the center point. Extension to the Gaussian shape laser is straightforward and the qualitative results will not be changed. When the laser is applied, a centripetal atom current will be induced and the current conservation condition will be violated at the center. In the 


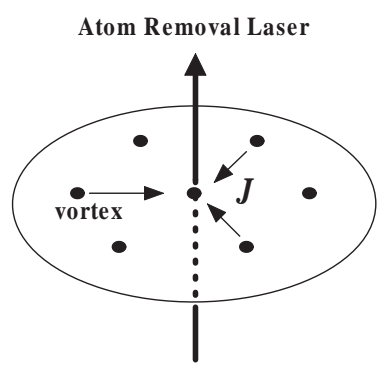

(a)

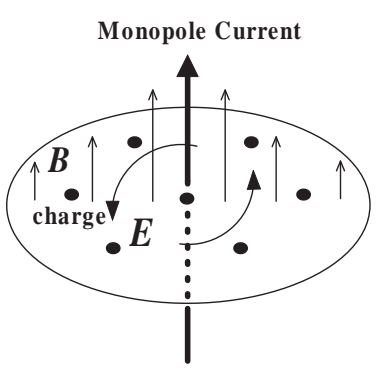

(b)

Figure 1. (a) Atom removal laser induces a current of atom in the original picture (b) Vortices are viewed as charges moving under a perpendicular magnetic field in the dual picture. The thin lines represent the magnetic and electric field, the thick lines represent the atom removal laser or monopole current.

dual picture, a circular electric field will be generated and the Maxwell equation should be modified in the following way

$$
\frac{\partial B}{\partial t}+\vec{\nabla} \times \vec{E}=I \delta(\vec{r}) .
$$

Here the value of $I$ equals to the number of atoms removed from the condensate per unit time. Therefore it is modelled as a monopole current running through the plane. The magnitude of the electric field is approximately $I /(2 \pi|\vec{r}|)$ nearby the center. For the case of continuous laser the vortices as charges will be accelerated azimuthally by the electric field, and the Lorentz force will drive the vortices to aggregate toward the center, and eventually to form a giant vortex.

\subsection{Two Kinds Oscillation}

After a pulse laser which only acts for a short time $\Delta T$, the vortices will acquire a velocity $\vec{v}$ and leave the balance positions of the lattice. For the vortices closest to the center, the magnitudes of their velocities can be approximated by

$$
v=E q \Delta T / m_{v} \simeq I \Delta T \hbar /\left(2 \pi m_{v} d\right),
$$

$d$ is the vortex lattice spacing. Therefore, there are two forces which can cause the oscillation motion, one is the Lorentz force $f_{1}$, and the other is the mutual interaction force between vortices $f_{2}$. It is easy to obtain the ratio of the two forces:

$$
\frac{f_{1}}{f_{2}}=I \Delta T \frac{m}{m_{v}} .
$$

For these experiments we can naturally assume that the number of atoms removed from the condensate $I$ is an increase function of the power of removal laser. Thus Eq. (15) indicates that the Lorentz force is dominant for a strong laser pulse, such as the pulse used in Ref. 11. In this limit, the vortices will execute circumnutation. When the vortices move very close to each other, they will merge into a giant vortex, and separate into several single vortices again when they oscillate away from each other. The mutual interaction force will be dominant for the weak pulse case. In this limit, it will cause a collective oscillation of vortex lattice known as Tkachenko oscillation. 
The oscillation frequency $\omega$ depends on the initial conditions of the condensate, such as the number of atoms and the rotation frequency $\Omega$. In the strong pulse limit, for the motion is mostly determined by the Lorentz force, $\omega=\hbar \rho / m_{v}$. Because the density $\rho$ is proportional to the number of atoms $N$ and is a decreasing function of the rotation frequency $\Omega$, the oscillation frequency is linearly dependent on $N$ and increases with $\Omega$ decreasing.

In the weak pulse limit, the oscillation frequency $\omega$ is proportional to $\sqrt{\rho} / d$. It is not obvious to tell whether $\omega$ will decrease or not when the condensate rotates faster, because both $\rho$ and $d$ will decrease with the rotation frequency $\Omega$ increasing. We make a rough estimate as following: For a fast rotating BEC confined in a harmonic trap with trapping frequency $\omega_{\perp}$, the effective confining potential is $\left(\omega_{\perp}^{2}-\Omega^{2}\right) r^{2} / 2$. Using the Thomas-Fermi approximation we can obtain that the condensate density nearby the center $\bar{\rho}$ is proportional to $\sqrt{1-\frac{\Omega^{2}}{\omega_{\perp}^{2}}}$, and then using the formula $d^{2} \propto 1 / \bar{\rho}_{v}=$ $\hbar /(2 m \Omega)$, we can obtain that

$$
\omega=C \sqrt{\frac{\Omega}{\omega_{\perp}} \sqrt{1-\frac{\Omega^{2}}{\omega_{\perp}^{2}}}} .
$$

Here $C$ is a complicated constant, which is related to the atom mass, vortex mass, trap frequency and the density distribution along the third spatial dimension. The Eq.(16) shows that $\omega$ is a decreasing function of $\Omega$ when $\Omega / \omega_{\perp}>1 / \sqrt{2}$, which is shown in the left side of Fig.(2) 14.

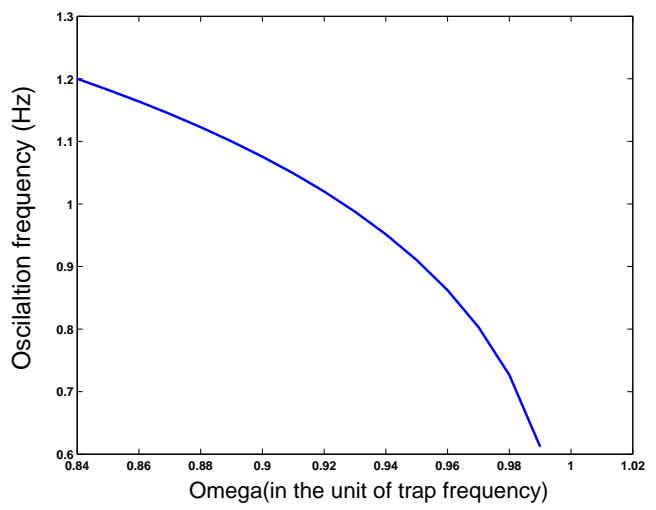

Figure 2. The oscillation frequency of vortices $\omega$ as a function of rotational frequency of condensate $\Omega$ (in the unit of $\omega_{\perp}$ ).

From above discussion we can see the atomic current toward the center is of essential importance for causing oscillations. Hence if there is any other ways to generate a similar atomic current, such as using optical dipole force to draw atoms into the middle of the condensate in the Ref.[2], the similar oscillation of vortex lattice was observed.

\subsection{Damping Effect}

One feature of these oscillation motions shown in these experiments is that the oscillation amplitudes are heavily damped, especially for the Tkachenko oscillation 


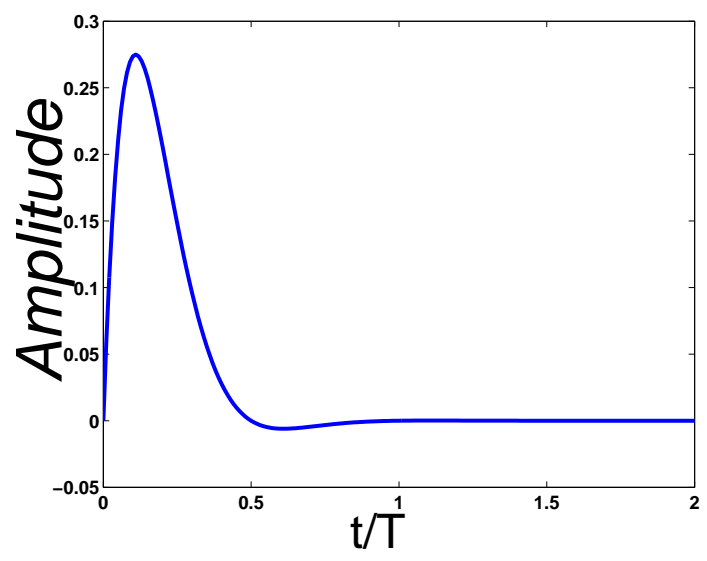

Figure 3. Heavily damped oscillation amplitude as a function of time $t$ (in the unit of oscillation period $T$ )

reported in Ref. [2]. It is quite natural in the view of the dual picture. Because the coupling between the vortex current and the phonon field is a gauge coupling, an accelerated vortex will radiate sound wave in the same way as an accelerated electron radiating electromagnetic wave. In the view of hydrodynamic picture, when a vortex is accelerating, the circular current $\vec{J}$, as well as the singularities of field $\vec{J}$, can not be stationary in any inertial frame. Due to the current conservation condition Eq.(4), a time dependent density fluctuation $\delta \rho(\vec{r}, t)$ will be generated. Hence the oscillating vortices will induce sound wave, lose their energy and result in the damping of amplitude. In the dual picture this process can be calculated through a standard MED calculation.

The retarded Green's function for $2+1 D$ MED is

$$
G\left(\tilde{r}, \tilde{r}^{\prime}\right)=\frac{\Theta\left(t-t^{\prime}\right)}{2 \pi} \frac{\Theta\left(s^{2}\left(t-t^{\prime}\right)^{2}-\left(\vec{r}-\vec{r}^{\prime}\right)^{2}\right)}{\sqrt{s^{2}\left(t-t^{\prime}\right)^{2}-\left(\vec{r}-\vec{r}^{\prime}\right)^{2}}} .
$$

And the gauge potential $\tilde{b}_{\text {rad }}$ for the radiation field is

$$
\tilde{b}_{\text {rad }}=\int_{-\infty}^{t-\left|\vec{r}-\vec{r}\left(t^{\prime}\right)\right| / s} d t^{\prime} \frac{\tilde{V}\left(t^{\prime}\right)}{\sqrt{s^{2}\left(t-t^{\prime}\right)^{2}-\left(\vec{r}-\vec{r}\left(t^{\prime}\right)\right)^{2}}} .
$$

Here $\tilde{V}\left(t^{\prime}\right)$ is the vortex 3 -current. Unlike the $3+1$ D case, the Green's function contains the step function $\Theta$ instead of Dirac's $\delta$ function, so one can not easily find a general expression for the radiation energy of a charge with arbitrary acceleration. 15] [16] Here we only consider two special cases, the linear oscillation $\vec{V}\left(t^{\prime}\right)=\left(v \cos \left(\omega t^{\prime}\right), 0\right)$, and circular motion $\vec{V}\left(t^{\prime}\right)=\left(-v \sin \left(\omega t^{\prime}\right), v \cos \left(\omega t^{\prime}\right)\right)$. Following the approximation made in Ref. [15], we replace $\left|\vec{r}-\vec{r}\left(t^{\prime}\right)\right|$ with $R$ and calculate the energy radiation along large circle $|r|=R$. Then we can perform an analytical calculation for the average energy radiated over one period,

$$
P=\frac{1}{T} \int_{0}^{T} \int_{0}^{2 \pi} d t d \theta r \vec{\sigma} \cdot \hat{r}
$$


where $\vec{\sigma}$ is the Poynting vector. For these two special cases, the result is

$$
P=\frac{\rho \hbar^{2} \omega v^{2}}{2 m s^{2}}=\frac{\hbar^{2} \omega v^{2}}{2 g} .
$$

The radiation rate is inverse proportional to the interaction strength $g$, so the damping effect is remarkable for the weak interacting boson gases. Recalling the approximate expression for the vortex mass $m_{v}=\bar{\rho} \hbar^{2} \ln (R / \Lambda) /\left(4 \pi s^{2} m\right)$, Eq.(20) can be reexpressed as

$$
P=\frac{1}{2} \frac{\rho \hbar^{2}}{s^{2} m} \omega v^{2}=\frac{1}{2} \frac{4 \pi \omega}{\ln \frac{R}{\Lambda}} m_{v} v^{2}=\frac{4 \pi \omega}{\ln \frac{R}{\Lambda}} E .
$$

Hence

$$
\frac{d E}{d t}=-\frac{4 \pi \omega}{\ln \frac{R}{\Lambda}} E,
$$

this indicates that the oscillation energy exponentially decays as $\exp \{-t / \tau\}$, and the ratio of the exponential damping time $\tau$ to the oscillation period $T$ is equal to $\ln \frac{R}{\Lambda} /\left(8 \pi^{2}\right)$. A typical oscillation curve in the current experiment conditions is schematically shown in the right side of Fig 3 one can see that the oscillation amplitude will decay rapidly within one period, and this is qualitatively coincident with the experimental observation [1] 2]. However, there are also a little quantitatively disagreement between the theoretical damping curve and the experiments, in Ref. [1] the amplitude vanishes within about three periods and in Ref. [2] it is about one and a half periods. This disagreement occurs because in the real case the vortex mass should be modified due to the spatial density inhomogeneity, the third spatial dimension effect and the edge effect. The mass of vortex, which is proportional to the self-energy, is therefore changed with the position of vortex. 17] It is expected that the damping curve will agree better with the experiments when these subtle effects are carefully considered.

\section{Summary}

According to the effective Maxwell electrodynamic model the vortices in the BEC can be viewed as an ensemble of classical charges moving in an external magnetic field. So far we have applied this description to understand the observed phenomena of vortices induced by selective removal atoms from a fast rotating BEC. The atom removal laser applied to the center of the condensate will cause two leading effects in the view of dual picture. One is the increase of the vortex chemical potential and then subsequently the increase of vortex density. The other is that it will induce a circular electric field, and each vortex will acquire an azimuthal velocity. We clarified that there are two forces that can cause oscillation motion, and discussed the the relation between the oscillation frequencies and the number of atoms together with the effective rotation frequency. We showed that the dispersion mechanism for the vortices oscillation comes from the emission of phonon, which is the same as the radiation of the electromagnetic wave of an accelerated charge. And the damping effect is more remarkable in these experiments because the interaction strength is weak.

In this paper the dual description of vortices has been used to explain current experiments. Although it is not yet a detailed quantitative description, it provides a natural physics picture of most key experimental observations, which convinces us to believe that this description is valid to the extent that the BEC is fast rotated but 
still not entering the quantum Hall mean field regime. We hope this will start a new route to study the issues in the rapidly rotating BEC system.

Acknowledgements: HZ would like to thank Professor C. N. Yang for encouragement. And the authors would like to acknowledge Z.Y. Weng, T.L. Ho, L. Chang, R. L $\ddot{u}$ and X.L. Qi for helpful discussions. This work is supported by National Natural Science Foundation of China ( Grant No. 10247002 )

\section{References}

[1] P. Engels, I. Coddington, P. C. Haljan, V. Schweikhard, E. A. Cornell, Phys. Rev. Lett 90, 170405 (2003)

[2] I. Coddington, P. Engels, V. Schweikhard, and E. A. Cornell, Phys. Rev. Lett 91, 100402 (2003)

[3] T.P.Simula, A.A.Penckwitt, and R.J.Ballagh, Phys. Rev. Lett, 92, 060401 (2004)

[4] G. Baym, Phys. Rev. Lett, 91, 110402 (2003)

[5] L.O.Baksmaty, S.J.Woo, S. Choi, and N.P.Bigelow, Phys. Rev.Lett, 92,160405 (2004) S. J. Woo, l.O. Baksmaty, S.Choi, and N.P. Bigelow, Phys. Rev. Lett, 92, 170402 (2004)

[6] M. Cozzini, L. P. Pitaevskii, and S. Stringari, Phys. Rev. Lett. 92, 220401 (2004)

[7] T. Mizushima, Y.Kawaguchi, K.Machida, T.Ohmi, T.Isoshima, and M.M. Salomaa, Phys. Rev. Lett, 92, 060407 (2004)

[8] R. Savit, Rev. Mod. Phys. 52, 453 (1980)

[9] V. N. Popov, Functional integrals and collective excitations, Cambridge University Press, (1987)

[10] D. H. Lee, M. P. A. Fisher Int. J. Mod. Phys 5, 2675 (1991); M. P. A. Fisher, D. H. Lee Phys. Rev. B 392756 (1989)

[11] D. P. Arovas and J. A. Freire, Phys. Rev. B 55, 1068 (1997)

[12] J. W. Negele and H. Orland, Quantum many-particle system, p. 66, Perseus Books Publishing (1988)

[13] T. L. Ho Phys. Rev. Lett. 87, 060403 (2001)

[14] We have made a rough estimate that the frequency given by equation 16 is the same order of the experiment date. And we use the experiment date $\Omega / \omega_{\perp}=0.84, \omega=1.2 \mathrm{~Hz}$ reported in Ref. 2 to determine the constant $C$

[15] E. Lundh and P. Ao, Phys. Rev. A 61, 063612 (2000)

[16] W. A. Moura-Melo and J. A. Helayël-Neto, Phys. Rev. D 63, 065013 (2001)

[17] M. Guilleumas and R. Graham, Phys. Rev. A 64, 033607 (2001) 